\title{
Assessment of the mechanisms exerting glucose-lowering effects of dried peas in glucose-intolerant rats
}

\author{
Kevin A. Whitlock ${ }^{1}$, Lisa Kozicky ${ }^{1}$, Alena $\mathrm{Jin}^{2}$, Homun Yee $^{1}$, Christine $\mathrm{Ha}^{2}$, Jill Morris ${ }^{2}$, \\ Catherine J. Field ${ }^{2}$, Rhonda C. Bell ${ }^{2}$, Jocelyn A. Ozga ${ }^{2}$ and Catherine B. Chan ${ }^{1,2 *}$ \\ ${ }^{1}$ Department of Physiology, University of Alberta, Edmonton, Alberta, Canada T6G $2 R 3$ \\ ${ }^{2}$ Department of Agricultural, Food and Nutritional Science, University of Alberta, Edmonton, Alberta, Canada T6G 2R3
}

(Submitted 31 August 2011 - Accepted 24 October 2011)

\section{Abstract}

The present study compared the effects of feeding uncooked pea fractions (embryo $v$. seed coat) on glucose homeostasis in glucoseintolerant rats and examined potential mechanisms influencing glucose homeostasis. Rats were made glucose intolerant by high-fat feeding, after which diets containing both high-fat and pea fractions were fed for 4 weeks. Rats fed diets containing uncooked pea seed coats low (non-coloured seed coat; NSC) or high (coloured seed coat; CSC) in proanthocyanidins but not embryos had improved oral glucose tolerance $(P<0 \cdot 05)$. NSC also lowered fasting and glucose-stimulated insulin secretion $(P<0 \cdot 05)$, decreased $\beta$-cell mass by $50 \%(P<0.05)$ and lowered levels of malondialdehyde, a marker of oxidative stress. Furthermore, NSC decreased the mucosal thickness of the colon by $25 \%(P<0 \cdot 05)$, which might affect fibre fermentation and other gut functions. Small but statistically significant $(P<0 \cdot 05)$ effects consistent with enhanced glucose transport or metabolism were observed in the skeletal muscle of rats fed NSC or CSC, for example, increased levels of AMP-dependent kinase or akt. We conclude that pea seed coats are the fraction exerting beneficial effects on glucose tolerance. Most of the changes were small in amplitude, suggesting that additive effects on multiple tissues may be important. NSC content appeared to have the most beneficial effects in improving glucose homeostasis but our ability to detect the effect of flavonoids may have been limited by their low concentration in the diet.

\section{Key words: Dried peas: Glucose homeostasis: Glucose tolerance: Diabetes: Rats}

Nutritional interventions are central to the treatment of type 2 diabetes. The 2008 Canadian Clinical Practice Guidelines for diabetes recommend increasing the intake of non-oil-seed pulses, including dried peas and beans, chickpeas and lentils $^{(1)}$. A recent meta-analysis of forty-one human randomised controlled trials concluded that pulse consumption predicts improved long-term glycaemic control ${ }^{(2)}$. Despite this conclusion, the heterogeneity among published studies precludes strong statements about the type, dose, duration of intake or mechanism of action. However, the effects of pulses are strongly correlated with the overall glycaemic index of the diet ${ }^{(2)}$, suggesting that the fibre and resistant starch components are important bioactive components.

Pulse seeds consist of an embryo surrounded by a seed coat. The embryo consists of cotyledons and an embryo axis, with the cotyledons making up about $98 \%$ of the embryo by weight in the pea (Pisum sativum L.) ${ }^{(3)}$. The embryo is mainly starch and protein with smaller quantities of soluble and insoluble fibre ${ }^{(4,5)}$. Uncooked field pea starch includes $2 \cdot 4 \%$ resistant $\operatorname{starch}^{(6)}$, which can be increased by cooking ${ }^{(7)}$, although this has not been reported in all studies even though digestibility decreased ${ }^{(6)}$. High fibre consumption $(50 \mathrm{~g} / \mathrm{d}$, with $25 \mathrm{~g}$ soluble fibre/d) is associated with reduced fasting plasma glucose, and reduced excursions of glucose and insulin postprandially ${ }^{(8)}$. Soluble fibre and resistant starch are fermented in the large intestine, producing SCFA, which may have multiple beneficial effects on metabolism. These are hypothesised to include modulation of hepatic glucose output ${ }^{(9)}$, induction of incretin secretion, and increasing insulin sensitivity ${ }^{(10)}$. However, there is conflicting evidence for these mechanisms in man and identification of mechanisms is limited by the methodology.

The pea seed coats consist mainly of insoluble fibre, and cultivars with clear seed coats have minimal flavonoid content (non-coloured seed coats; NSC), while other cultivars contain polyphenolic flavonoid compounds such as proanthocyanidins, which upon oxidation appear brown (coloured

Abbreviations: AMPK, AMP-dependent kinase; CSC, coloured seed coat; HFD, high-fat diet; MDA, malondialdehyde; NSC, non-coloured seed coat; NT, nitrotyrosine; PEPCK, phosphoenol pyruvate carboxykinase; SGLT1, Na-glucose linked transporter-1. 
seed coats; CSC) ${ }^{(11)}$. Flavonoids are abundant in many fruits, legumes and nuts but not grains, and are of interest nutritionally for their antioxidant properties ${ }^{(12)}$. For example, grapes are rich in flavonoids and their consumption is associated with reduced risk of cardiovascular and other chronic diseases $^{(13)}$. Less is known about proanthocyanidins and their effects on type 2 diabetes. Most, but not all, studies of consumption of proanthocyanidins derived from grapes suggest that glycaemic control is improved ${ }^{(14)}$. One study of $d b / d b$ mice found that persimmon peel-derived proanthocyanidins given orally had both hypoglycaemic and hypolipidaemic effects $^{(15)}$. Proanthocyanidins may also delay glucose absorption from the intestine, improve $\beta$-cell function and mimic the effects of insulin on target tissues ${ }^{(12)}$

Differences in composition suggest that consumption of different pea fractions may elicit different metabolic effects. In a type 2 diabetes population, a mixed meal including whole dried peas results in smaller postprandial increases in glucose and insulin compared with potatoes ${ }^{(16)}$. Muffins made from whole pea flour reduced fasting insulin and improved insulin sensitivity but did not reduce the postprandial glycaemic response of overweight subjects compared with wheat flour ${ }^{(17)}$. However, diets containing pea seed coats may elicit different effects from embryos or whole peas. Pure pea starch elicited lower post-meal glucose and insulin excursions than maize $\operatorname{starch}^{(18)}$. Isolated pea fibre added to a meal produced a lower postprandial insulin response when compared with low-fibre test meals in young, healthy subjects $^{(19)}$. Fasting insulin but not the postprandial glucose excursion was reduced when ground pea fibre was substituted for wheat flour $(22 \%, \mathrm{w} / \mathrm{w})$ in muffins ${ }^{(17)}$.

To a large extent, the experimental designs of these studies does not allow for elucidation of mechanisms and it is thus not understood where pulses, such as peas, predominantly exert their effects on the gastrointestinal tract, on insulin-sensitive tissues such as muscle or on insulin-secreting $\beta$-cells. Furthermore, it is unclear which of the pulse components (seed coat $v$. embryo) has the most beneficial effects, and whether pulses with high levels of antioxidants such as proanthocyanidins exert different effects than those without. The overall goal of the present study is to understand the metabolic and molecular basis for the beneficial effects of peas in the diet on glucose homeostasis.

\section{Methods and materials}

\section{Pea seed analysis}

The pea seed cultivars 'Courier' and 'Canstar' used in the present study were grown in western Canada in 2007. Total phenolic content and antioxidant activities of legumes are correlated with the colour of the seed coat ${ }^{(11)}$. The seed coats of 'Courier' are brownish in colour (CSC). For protein analysis, 5-10 g of mature seeds, seed coats or embryos were ground to a powder and protein was determined from subsamples of this tissue according to the AOAC method $972.43^{(20)}$ using 6.25 as the conversion factor for calculating protein content (\%). For fibre analysis, 5-10g of mature seeds, seed coats or embryos were ground to a powder and subsamples of these tissues were used to determine the acid-detergent fibre component according to the ANKOM Technology Method $6^{(21)}$ and the neutral-detergent fibre component according to the ANKOM Technology Method $5^{(22)}$. Total proanthocyanidin content values were based on characterisation of proanthocyanidin subunits following phloroglucinolysis and reverse phase-high pressure liquid chromatography-diode array detector analysis as described by Kennedy \& Jones ${ }^{(23)}$.

The pea seeds were mechanically dehulled using a dehuller (Forsberg model 2; Forsberg) and separated into embryo and seed coat fractions using an aspirator (Carter Duo). The embryos ('Courier' only) and seed coats were then ground to a fine consistency for inclusion in the diets. Fine seed coats were obtained via sieving with a no. 16 Canadian standard sieve.

\section{Experimental animals and diets}

All animal protocols followed the guidelines of the Canadian Council on Animal Care and were approved by the Health Sciences Animal Care and Use Committee at the University of Alberta. Sprague-Dawley rats were obtained from Charles River Canada at 8 weeks of age. After 1-week acclimatisation with access to standard chow and water ad libitum, all rats were introduced to a high-fat diet (HFD, $40 \%$ of energy from fat, $20 \%$ of energy from saturated fat) composed as shown in Table 1. At 3 weeks later, diets with uncooked pea fractions added to the base diet were introduced and fed for 4 weeks. Embryos of pea varieties have similar protein composition but can vary widely (up to $7 \%$ in one study) within cultivars over several growing years, depending on agronomic and environmental conditions ${ }^{(24)}$. Thus, only embryos from 'Courier' peas were used in the present study and total protein was adjusted to be equal in both diets. Composition of the diets containing pea embryos or pea seed coats is shown in Table 1. Diets were formulated to ensure equal total fat (20\%), protein (27\%), carbohydrate (38\%) and fibre (8\%) and thus were equal in energy density.

\section{Glucose tolerance tests}

Glucose tolerance tests were conducted at baseline, and at 3 and 7 weeks (i.e. before HFD, after 3 weeks of HFD and after 4 weeks of HFD plus pea fractions). At the same time, rats were weighed and food and water intake measured over a $24 \mathrm{~h}$ period. Rats were acclimatised to individual metabolism cages for $24 \mathrm{~h}$ before food and water intakes were determined. Oral glucose tolerance tests were performed after an overnight fast essentially as described ${ }^{(25)}$ except that glucose was measured in whole blood with a glucometer (Accu-Check Compact Plus; Roche Diagnostics) over a $2 \mathrm{~h}$ period. Additional $50 \mu \mathrm{l}$ blood samples were centrifuged and the serum collected and stored at $-80^{\circ} \mathrm{C}$ until assayed for insulin using an ELISA, according to the manufacturer's instructions (Alpco). 
Table 1. Diet composition $(\mathrm{g} / \mathrm{kg})^{*}$

\begin{tabular}{|c|c|c|c|c|}
\hline Diet group... & HFD & EMB & NSC & CSC \\
\hline Casein & 270 & 244 & $267 \cdot 2$ & $264 \cdot 1$ \\
\hline Maize starch $\ddagger$ & 189 & 189 & 189 & 189 \\
\hline Dextrose† & 189 & 189 & 189 & 189 \\
\hline Cellulose† & $80 \cdot 0$ & $74 \cdot 7$ & $41 \cdot 0$ & $41 \cdot 0$ \\
\hline Rapeseed stearine§ & 99.5 & $99 \cdot 5$ & 99.5 & 99.5 \\
\hline Sunflower-seed oilł & 94.5 & 94.5 & 94.5 & 94.5 \\
\hline Flaxseed oill & $6 \cdot 0$ & $6 \cdot 0$ & $6 \cdot 0$ & $6 \cdot 0$ \\
\hline Vitamin mix AIN-93-VX† & $10 \cdot 0$ & $10 \cdot 0$ & $10 \cdot 0$ & $10 \cdot 0$ \\
\hline Mineral mix Bernhart \& Tomarelli† & $51 \cdot 0$ & $51 \cdot 0$ & $51 \cdot 0$ & $51 \cdot 0$ \\
\hline DL-Methionine & 2.5 & 2.5 & 2.5 & 2.5 \\
\hline Choline chloride & $2 \cdot 8$ & $2 \cdot 8$ & $2 \cdot 8$ & $2 \cdot 8$ \\
\hline Inositolq & $6 \cdot 3$ & $6 \cdot 3$ & $6 \cdot 3$ & $6 \cdot 3$ \\
\hline Pea embryo & 0 & 100 & 0 & 0 \\
\hline Pea seed coat & 0 & 0 & $56 \cdot 5$ & 75 \\
\hline Total weight $(\mathrm{g})$ & $1000 \cdot 6$ & $1069 \cdot 3$ & $1015 \cdot 3$ & $1030 \cdot 7$ \\
\hline Carbohydrate (\%) & $37 \cdot 7$ & $36 \cdot 6$ & $37 \cdot 2$ & $36 \cdot 7$ \\
\hline Fat $(\%)$ & $20 \cdot 0$ & $19 \cdot 4$ & $19 \cdot 7$ & $19 \cdot 4$ \\
\hline Protein (\%) & $27 \cdot 0$ & $26 \cdot 2$ & $26 \cdot 6$ & $26 \cdot 2$ \\
\hline Fibre (\%) & $8 \cdot 0$ & $7 \cdot 8$ & 7.9 & $7 \cdot 8$ \\
\hline \multicolumn{5}{|c|}{$\begin{array}{l}\text { HFD, high-fat diet; EMB, diet supplemented with pea embryos from 'Courier' peas; NSC, diet } \\
\text { supplemented with non-coloured seed coats; CSC, diet supplemented with coloured seed } \\
\text { coats; AIN, American Institute of Nutrition. }\end{array}$} \\
\hline $\begin{array}{l}\text { * See Table } 2 \text { for protein and fibre ana } \\
\text { added in an amount calculated to rep } \\
\text { were added in an amount to provide a } \\
\text { † Harlan. } \\
\text { † Canada Safeway. } \\
\text { § Richardson Oilseed. } \\
\text { ॥ Shoppers Drug Mart. } \\
\text { ๆ MP Biomedicals. }\end{array}$ & $\begin{array}{l}\text { S of the p } \\
\text { ent } 10 \% \text { o } \\
\text { oximately } h\end{array}$ & $\begin{array}{l}\text { seed fracti } \\
\text { tal carbohy } \\
\text { the total fib }\end{array}$ & $\begin{array}{l}\text { The pea } \\
\text { te. The pe } \\
\text { the diet. }\end{array}$ & oryo was \\
\hline
\end{tabular}

\section{Tissue collection}

At $5 \mathrm{~d}$ following the glucose tolerance test, rats were fasted overnight and then euthanised under anaesthesia (ketamine $100 \mathrm{mg} / \mathrm{kg}$ and xylazine $1 \mathrm{mg} / \mathrm{kg}$, intraperitoneally) by exsanguination. Half the rats received insulin $(35 \mathrm{pmol} / \mathrm{kg}$ intraperitoneally) $15 \mathrm{~min}$ before death while the other half received an equivalent volume of saline. Blood $(5-10 \mathrm{ml})$ was obtained from the abdominal aorta, and divided for preparation of plasma and serum, which were frozen at $-80^{\circ} \mathrm{C}$. Samples of liver, jejunum, ascending colon, soleus and epitrochearis muscle were snap-frozen in liquid $\mathrm{N}_{2}$ and stored at $-80^{\circ} \mathrm{C}$. An additional pancreas sample just adjacent to the spleen was fixed in formalin overnight, then transferred to $70 \%$ ethanol in preparation for embedding in paraffin by standard techniques. Jejunal and colonic samples were similarly fixed and prepared for histology.

\section{Analysis of serum and plasma samples}

Fasting serum TAG (Trinder, Sigma), total cholesterol and NEFA were obtained from the baseline sample using kits (Wako Chemicals USA). Haptoglobin in serum was measured using a kit from Tridelta.

\section{Immunoblotting}

Frozen samples were thawed on ice and homogenised in LIPA buffer as described ${ }^{(26)}$. Total protein was measured using a Lowry protein assay (Sigma). Samples $(20 \mu \mathrm{g}$ protein) were subjected to SDS-PAGE followed by blotting onto nitrocellulose membranes. The membranes were blocked in 5\% defatted skimmed milk-1\% Tween-PBS for $60 \mathrm{~min}$ followed by overnight incubation at $4^{\circ} \mathrm{C}$ with primary antibodies diluted in Tris-buffered saline-1\% Tween-5\% bovine serum albumin. Antibodies from Cellular Signaling Technology included phospho-Thr172-AMP-dependent kinase (AMPK) $\alpha$-subunit (1:1000), AMPK $\alpha$-subunit (1:1000), phospho-Ser473-akt (1:1500), akt (1:1500) and GLUT4 (1:2000). Anti-phosphoenol pyruvate carboxykinase (PEPCK; 1:1000) was from Cayman Chemicals. Membranes were subsequently incubated with the appropriate peroxidase-conjugated secondary antibodies (Sigma) for $1-2 \mathrm{~h}$ at room temperature. Membranes were developed using ECL Plus (GE Biosciences) and digital images captured on a Typhoon Trio imaging station (GE Healthcare). Labelling of specific proteins was compared with $\beta$-actin (liver; Sigma) or $\beta$-tubulin or glyceraldehyde-3phosphate dehydrogenase (GAPDH) (muscle; Abcam) used as loading controls using ImageJ (http:///rsb.info.nih.gov/ij).

\section{Histology, immunohistochemistry and morphometry}

Before embedding, gut sections were cut into five or six rings and oriented in the paraffin so as to obtain cross-sections. Paraffin-embedded pancreatic or gut tissue was sectioned $(5 \mu \mathrm{m})$ and serial sections mounted on glass slides. After dewaxing in xylene and graded alcohol, slides were rinsed with distilled water and PBS. For characterisation of gut morphology, the tissues were stained with haematoxylin and eosin and digital photomicrographs acquired using an Axiovert 
Table 2. Protein and fibre analysis of whole seeds, seed coats and embryos of pea cultivars 'Courier' and 'Canstar' (Mean values with their standard errors of three replicates)

\begin{tabular}{|c|c|c|c|c|c|c|c|c|}
\hline \multirow[b]{2}{*}{ Pea (Pisum sativum L.) } & \multicolumn{2}{|c|}{ PA (\% fresh wt) } & \multicolumn{2}{|c|}{ Protein* (\% dry wt) } & \multicolumn{2}{|c|}{ ADF† (\% dry wt) } & \multicolumn{2}{|c|}{ NDF $\ddagger$ (\% dry wt) } \\
\hline & Mean & SE & Mean & SE & Mean & SE & Mean & SE \\
\hline 'Courier' whole seed & & & $24 \cdot 36$ & 0.69 & 7.90 & 0.82 & $12 \cdot 06$ & $1 \cdot 12$ \\
\hline 'Canstar' whole seed & & & $21 \cdot 86$ & 0.28 & $7 \cdot 75$ & 0.16 & 13.65 & $1 \cdot 16$ \\
\hline ‘Courier' seed coat & 4.40 & 0.09 & 7.92 & 0.37 & $42 \cdot 75$ & 0.53 & $52 \cdot 24$ & 0.64 \\
\hline 'Canstar' seed coat & $\mathrm{Nd}$ & & 4.97 & 0.17 & 58.84 & 0.24 & 68.98 & 0.12 \\
\hline ‘Courier’ embryo & & & 26.03 & 0.16 & 1.69 & 0.16 & $5 \cdot 27$ & 0.25 \\
\hline 'Canstar' embryo & & & $23 \cdot 36$ & 0.61 & 1.06 & 0.14 & 6.33 & 0.71 \\
\hline
\end{tabular}

PA, proanthocyanidins (flavonoids); ADF, acid-detergent fibre; NDF, neutral-detergent fibre; nd, not detected.

* Determined using AOAC method $972.43^{(20)}$.

†ADF is the residue remaining after digesting with $\mathrm{H}_{2} \mathrm{SO}_{4}$ and cetyltrimethylammonium bromide (CTAB). The fibre residues are predominantly cellulose and lignin. ANKOM Technology Method $5^{(22)}$.

$\ddagger$ NDF is the residue remaining after digesting in a detergent solution. The fibre residues are predominantly hemicelluloses, cellulose and lignin. ANKOM Technology Method $6^{(21)}$.

microscope and Axiovision 4.7 software (Carl Zeiss Canada Ltd). Villus height or mucosal thickness was quantified using Image J software, by drawing a line at right angles to the serosa. Only images where good cross-sectional orientation had been achieved were used and at least ten well-oriented samples were taken for each slide. Immunohistochemistry was performed to detect GLUT2, GLUT5 and Na-glucose linked transporter-1 (SGLT1). Sections were blocked in appropriate normal serum $(4 \%, 1 \mathrm{~h}$, room temperature), then primary antibodies (all 1:000; all from Millipore) for $1 \mathrm{~h}$ at room temperature. Appropriate fluorescent (AlexaFluor 488)-labelled secondary antibodies (Invitrogen) were applied for 2-4 h at 1:2000 (against GLUT5) or 1:4000 (against GLUT2 and SGLT1).

For determination of $\alpha$ - and $\beta$-cell areas, endogenous peroxidases were blocked with $3 \% \mathrm{H}_{2} \mathrm{O}_{2}$ for $30 \mathrm{~min}$. After washing in PBS, the mounted tissue was incubated in 5\% normal sheep serum for $20 \mathrm{~min}$ at room temperature, then blotted, and the primary antibody applied. Primary antibodies were mouse anti-glucagon (1:500; Abcam) and mouse antiinsulin (1:100; Dako). Slides were incubated overnight at $4{ }^{\circ} \mathrm{C}$ in a humidified chamber, then washed and incubated with peroxidase-conjugated sheep anti-mouse antibody (1:200) for $1 \mathrm{~h}$ at room temperature. Positive immunoreactivity was visualised by incubating the slides in diaminobenzidine plus $\mathrm{H}_{2} \mathrm{O}_{2}$ for $10 \mathrm{~min}$. Slides were then dehydrated in graded alcohol, cleared in xylene and cover-slipped. The entire section on each slide was photographed using an Axiovert microscope equipped with Axiovision 4.7 software (Zeiss). The total pancreatic area (excluding large ducts and veins), and the insulin- and glucagon-positive areas were quantified using Image as described ${ }^{(26)}$.

Oxidative stress was assessed in pancreatic islets by staining for either malondialdehyde (MDA) or nitrotyrosine (NT). To determine if the HFD increased MDA and NT staining, samples obtained from age-matched chow-fed Sprague-Dawley rats were obtained from a slide bank for comparison. For MDA, slides were blocked with 5\% normal rabbit serum, then incubated overnight at $4{ }^{\circ} \mathrm{C}$ with anti-MDA antiserum (1:100; Ray Biotech). For NT, slides were blocked with 5\% normal goat serum followed by anti-NT antiserum (1:100; Millipore).

Table 3. Metabolic profile of rats fed diets containing pea components

(Mean values with their standard errors of eight replicates)

\begin{tabular}{|c|c|c|c|c|c|c|c|c|}
\hline \multirow[t]{2}{*}{ Diet group... } & \multicolumn{2}{|c|}{ HFD } & \multicolumn{2}{|c|}{ EMB } & \multicolumn{2}{|c|}{ NCS } & \multicolumn{2}{|c|}{$\mathrm{csc}$} \\
\hline & Mean & SE & Mean & SE & Mean & SE & Mean & SE \\
\hline \multicolumn{9}{|l|}{ Body weight (g) } \\
\hline Baseline & $421 \cdot 1$ & 5.4 & $438 \cdot 8$ & $9 \cdot 2$ & $421 \cdot 8$ & $15 \cdot 6$ & $414 \cdot 1$ & $5 \cdot 8$ \\
\hline Final & $585 \cdot 2$ & $12 \cdot 1$ & $595 \cdot 8$ & $15 \cdot 3$ & $575 \cdot 0$ & 9.4 & $569 \cdot 6$ & 19.4 \\
\hline Change & $168 \cdot 8$ & $8 \cdot 6$ & $157 \cdot 0$ & $10 \cdot 0$ & $160 \cdot 1$ & 9.5 & $161 \cdot 3$ & $11 \cdot 3$ \\
\hline Fasting blood glucose $(\mathrm{mm}) \ddagger$ & $5 \cdot 3$ & 0.4 & $5 \cdot 8$ & 0.2 & $4 \cdot 4^{*}+$ & 0.2 & $4 \cdot 8^{*} \dagger$ & 0.2 \\
\hline Fasting serum insulin (рм)‡ & $189 \cdot 3$ & $68 \cdot 8$ & 258.2 & 51.6 & $68 \cdot 8^{\star *}$ & 34.4 & 258.2 & $86 \cdot 1$ \\
\hline Serum TAG $(\mathrm{mm}) \S$ & 1.72 & 0.21 & 1.89 & 0.29 & 1.73 & 0.17 & 1.84 & 0.22 \\
\hline Serum NEFA $(\mathrm{mm}) \S$ & 1.69 & 0.08 & 1.55 & 0.12 & 1.57 & 0.09 & 1.88 & 0.14 \\
\hline Total plasma cholesterol $(\mathrm{mm}) \S$ & $29 \cdot 7$ & 1.0 & $30 \cdot 8$ & $1 \cdot 2$ & 30.54 & $2 \cdot 2$ & 31.4 & 0.9 \\
\hline Serum haptoglobin $(\mathrm{mg} / \mathrm{ml}) \S$ & $1 \cdot 1$ & $0 \cdot 15$ & $1 \cdot 1$ & 0.14 & $1 \cdot 1$ & $0 \cdot 16$ & $1 \cdot 1$ & 0.27 \\
\hline
\end{tabular}

HFD, high-fat diet; EMB, diet supplemented with pea embryos; NSC, diet supplemented with non-coloured seed coats; CSC, diet supplemented with coloured seed coats.

Mean value was significantly different from that of the EMB group: ${ }^{*} P<0.05,{ }^{* *} P<0.01$.

† Mean value was significantly different from that of the HFD group $(P<0.05)$.

$\ddagger$ Blood sampling was done at completion of the diet feeding period during the oral glucose tolerance test.

$\S$ Serum for lipids and haptoglobin was harvested from blood collected from anaesthetised animals just before euthanasia. 
Appropriate secondary antibodies conjugated to Alexa-546 were used (1:200, room temperature, $1 \mathrm{~h}$; Invitrogen) to visualise antigen localisation. Dual or serial-section labelling was accomplished by applying the antibody against insulin (1:1000; Dako) followed by the appropriate secondary antibody conjugated with Alexa-488.

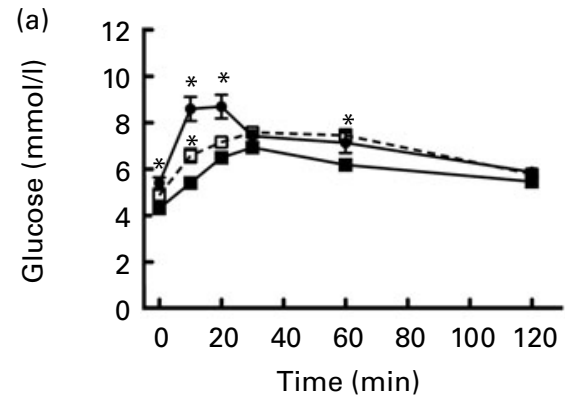

(c)
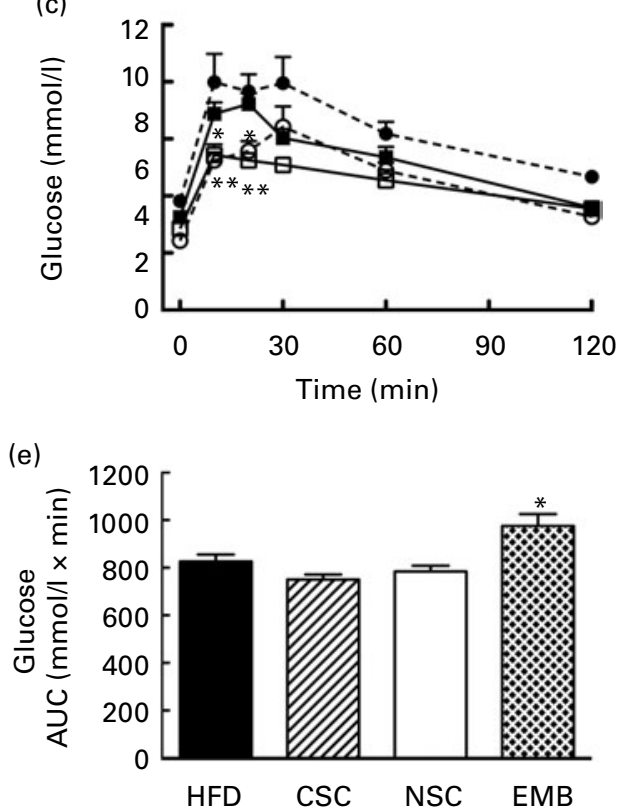

\section{Statistical analysis}

Data were expressed as mean values with their standard errors. Differences between groups were assessed by oneway or two-way ANOVA, as appropriate, and $P<0.05$ was considered significant. Specific differences were identified by the Newman-Keuls post hoc test.

(b)

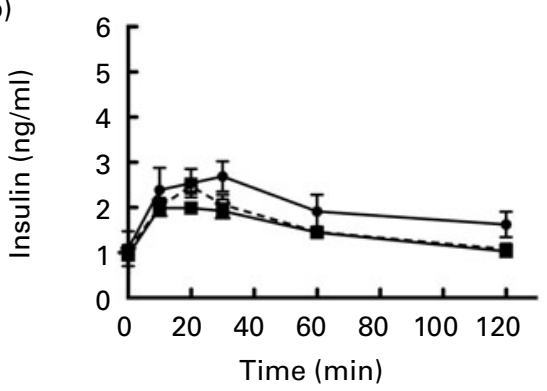

(d)

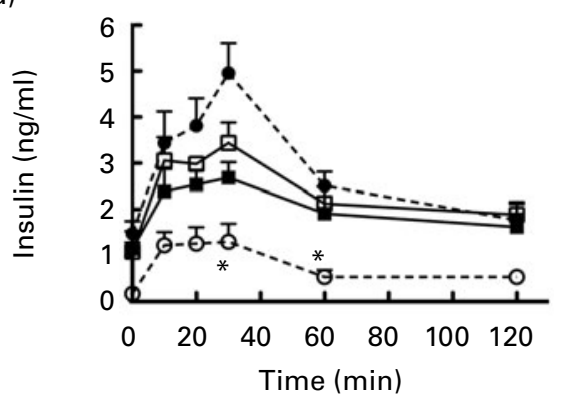

(f)

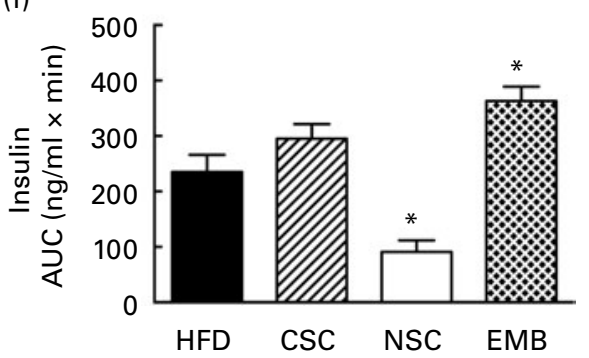

(g)

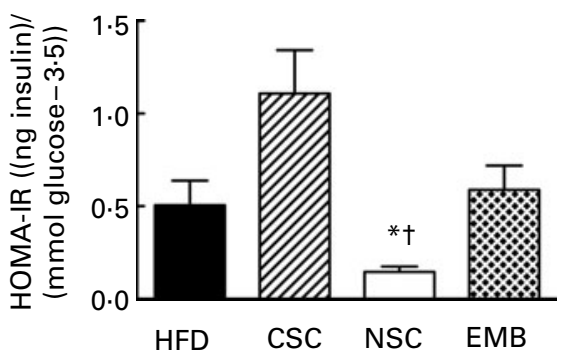

Fig. 1. Effects of feeding pea fractions on oral glucose tolerance in rats. Effect of 3 weeks $(-\square-)$ and 7 weeks ( $\bullet-)$ of feeding a $40 \%$ (w/w) fat diet (high-fat diet; HFD) on oral glucose tolerance (a) and insulin secretion (b) in response to administration of $1 \mathrm{~g} / \mathrm{kg}$ oral glucose. ( - -), Baseline. Values are means, with standard errors represented by vertical bars. *Mean value was significantly different from that at baseline $(P<0.05)$. (c) Blood glucose and (d) plasma insulin responses to $1 \mathrm{~g} / \mathrm{kg}$ oral glucose in rats on the HFD for 3 weeks, then continued on the HFD $\left(-\frac{-}{n} n\right.$ 16) or switched to the HFD plus coloured seed coats (CSC; $-\square-; n 16)$, non-coloured seed coats (NSC; $-0-; n 16)$ or embryos (EMB; $--; n 8)$ for 4 weeks. Values are means, with standard errors represented by vertical bars. Mean value was significantly different from that of the HFD group: ${ }^{*} P<0.05,{ }^{*} P<0.01$. (e, f) Incremental areas under the curve (AUC) for the glucose tolerance and insulin secretion data shown in (c) and (d). Values are means, with standard errors represented by vertical bars. *Mean value was significantly different from that of the HFD group $(P<0.05)$. (g) Calculation of insulin sensitivity using the homeostasis model of insulin resistance (HOMA-IR). Values are means, with standard errors represented by vertical bars. ${ }^{*}$ Mean value was significantly different from that of the HFD group $(P<0.05)$. †Mean value was significantly different from that of the CSC group $(P<0.05)$. 


\section{Results}

\section{Pea embryo and seed coat fraction analysis}

The seed coat component of the pea seeds was high in fibre (approximately 50 to $70 \%$ by weight), with 'Canstar' seed coats (NSC) having a higher fibre content than that of 'Courier' (CSC) (Table 2). Approximately 10\% of the seed coat fibre component was estimated to be hemicellosic in both cultivars (the difference between acid-detergent fibre and neutral-detergent fibre values; Table 2). The protein in the seed coats ranged from 5 to $8 \%$ by weight. The proanthocyanidin content was approximately $4 \%$ of fresh weight of CSC but not detectable in NSC fractions. In the final diet preparation, the proanthocyanidin content was $0.3 \%$. The protein levels in the embryos of both cultivars ranged from 23 to $26 \%$ by weight (Table 2). Although protein content was higher in the 'Courier' peas, the difference fell inside the range of variability expected within a cultivar. Therefore, the rat feeding trials were conducted using only 'Courier' pea embryos.

\section{Food intake}

Dietary consumption was measured at 7 weeks over $24 \mathrm{~h}$ while the rats were singly housed in a metabolism chamber. No differences in food intake were observed in the rats fed the HFD (26.0 (sE 1.6) g) compared with rats fed the HFD + embryos (27.0 (SE 1.9) g), HFD + NSC (28.6 (SE 1.4) g) or HFD + CSC (26.9 (sE 1.7) g). This suggested that palatability of the diets or altered satiation did not affect the outcome.

\section{Metabolic profile}

The metabolic profile of the rats is shown in Table 3. Body weight was compared at baseline and at study completion. The increase in body weight over the 7-week study was approximately $160 \mathrm{~g}$ and did not differ between groups $(P>0.05)$. Fasting blood glucose of both NSC and CSC groups was lower than in the embryo group, and that in the NSC group was also lower than in the HFD group $(P<0 \cdot 05)$. Fasting serum insulin concentration was lower in the NSC group than in any other group $(P<0 \cdot 01)$. Blood lipids, including TAG, NEFA and total cholesterol did not differ among diet treatments. Serum haptoglobin, a marker of inflammation, was similar in all groups.

\section{Oral glucose tolerance}

To quantify the degree of glucose intolerance induced by feeding the HFD, oral glucose tolerance was measured at baseline, and at 3 and 7 weeks. As shown in Fig. 1(a), the glucose excursion increased with the diet duration $(F(2,5)=27.57 ; P<0.0001)$, with significantly more glucose in the blood at $10 \mathrm{~min}$ in the animals fed the HFD for 3 weeks and at 0,10 and $20 \mathrm{~min}$ in the animals fed the HFD for 7 weeks $(P<0 \cdot 05)$. The insulin response to glucose after the HFD was also higher than at baseline $(F(2,5)=4 \cdot 203$; $P<0 \cdot 02$ ) (Fig. 1(b))
After 7 weeks ( 4 weeks fed pea fractions), the effects of the four diets on oral glucose tolerance were compared. Data are shown as the responses at each time point (Fig. 1(c)) and as incremental areas under the curve (Fig. 1(e)). The diet had a significant effect on oral glucose tolerance $(F(3,5)=11 \cdot 93$; $P<0.001)$ and the embryo group had an overall higher glucose excursion (Fig. 1(e)). Both the CSC and NSC groups had lower blood glucose than controls at 10 and $20 \mathrm{~min}$. Insulin responses also differed as a result of the diets $(F(3,5)=11 \cdot 01 ; \quad P<0 \cdot 001 ;$ Fig. $1(\mathrm{~d}))$, with the total response of the NSC group lower than any other group, and the embryo group higher than controls (Fig. 1(f)). The homeostasis model of insulin resistance (HOMA-IR) is a measure of insulin resistance based on fasting insulin and glucose concentrations. NSC-treated rats were less insulin resistant than HFD control or CSC rats using this calculation $(P<0 \cdot 05)$.

(a)
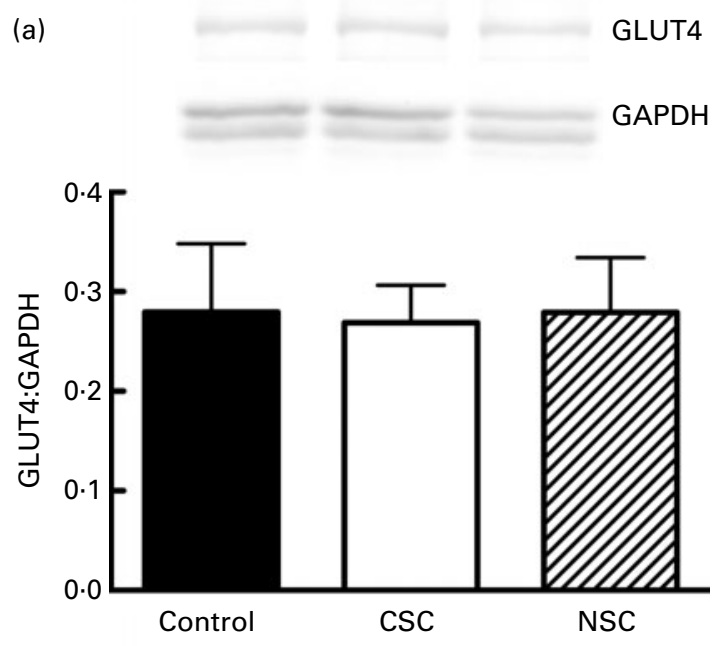

(b)

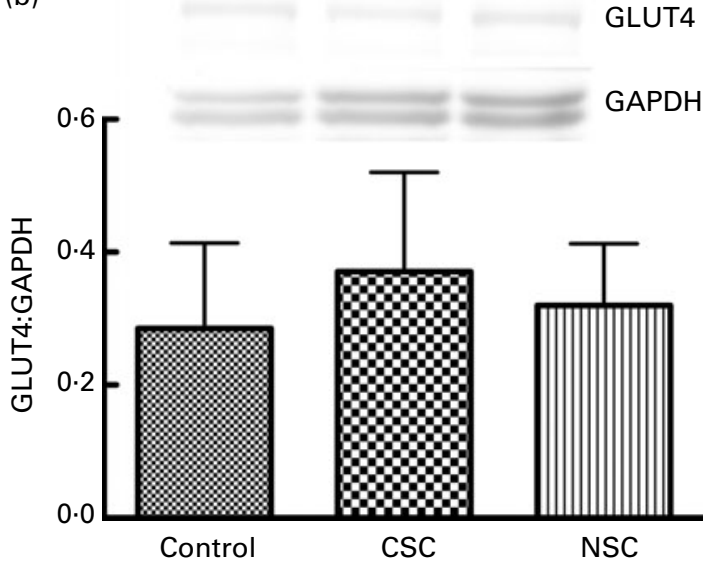

Fig. 2. Effects of feeding pea seed coats on GLUT4 levels in muscle measured by immunoblotting. GLUT4 protein relative to glyceraldehyde-3phosphate dehydrogenase (GAPDH) in rats fed a high-fat diet alone (control; $n 8$ ) or supplemented with coloured seed coats $(n 8)$ or non-coloured seed coats $(n 8)$ in (a) soleus and (b) epitrochlearis muscle lysates. Values are means, with standard errors represented by vertical bars. Insets show representative blots. 


\section{Insulin signalling pathways in skeletal muscle and liver}

Improved oral glucose tolerance could result from enhanced insulin sensitivity in peripheral tissues. We measured total GLUT4 protein levels in skeletal muscle from HFD, NSC and CSC rat groups. No differences $(P>0.05)$ between the groups were detected in either highly oxidative (soleus) muscle (Fig. 2(a)) or epitrochlearis muscle, which is mainly glycolytic (Fig. 2(b)).

To determine if up-regulation of the insulin-signalling pathway regulating glucose transport had occurred, phospho-akt and total akt were measured. Total akt protein expression was increased by $80 \%$ in epitrochlearis muscle of the CSC group $(P<0.05$; Table 4$)$. The relative phosphorylation was similar in all groups. No differences in akt or phospho-akt were detected in the soleus muscle or liver.

AMPK-regulated glucose uptake might also contribute to altered fasting glucose or glucose tolerance. In the soleus muscle of NSC rats, total AMPK protein expression was $2 \cdot 1$-fold greater than in HFD rats $(P<0 \cdot 05)$. There were no differences in the relative phospho-AMPK levels. In the liver of NSC-fed rats, total AMPK expression was reduced by about $60 \%(P<0.05)$. The amount of PEPCK was also analysed in the liver but no differences were observed between the groups. Data are summarised in Table 4.

\section{Pancreatic $\beta$-cells and oxidative stress}

Lower fasting insulin and a lower insulin response to glucose in the NSC group suggested that the number of $\beta$-cells contributing to secretion might be reduced. The area positively immunostained for insulin was quantified in pancreas sections from all four groups and compared with the total pancreatic area. As shown in Fig. 3(a), the $\beta$-cell area in the NCS group was decreased by about $70 \%$ compared with HFD controls $(F(3,21)=5 \cdot 167 ; P<0 \cdot 01) . \alpha$-Cell area (as assessed by glucagon staining) did not differ between the groups (Fig. 3(b)).

To evaluate whether islets from the pea-fed groups exhibited different patterns of oxidative stress, pancreatic sections were stained for MDA or NT and compared with insulinimmunoreactive cells. MDA was predominantly detected in cells co-staining for insulin, including a chow-fed (low-fat diet) control (Fig. 4(a)). In $\beta$-cells, staining was least intense in the NSC and embryo tissue when all slides were photographed under identical parameters. NT was detected in non- $\beta$-cells of all groups (Fig. 4(b)) but diet-related changes in staining intensity were not as pronounced as for MDA.

\section{Gut morphology}

No differences were detected in the height of villi in the jejunum (Fig. 5(a)) but the thickness of the colonic mucosa was significantly reduced in NSC-fed rats (Fig. 5(b)). In all groups, GLUT2, GLUT5 and SGLT1 were detected by immunostaining in both the jejunum and ileum but overt differences in staining intensity were not observed (not shown).

\section{Discussion}

Uncooked CSC and NSC from dried peas alleviated glucose intolerance in glucose-intolerant rats whereas embryos did not have beneficial effects. Lack of effect of the embryos may be attributed to the method of preparation. Uncooked peas have a lower content of amylase-resistant starch and

Table 4. Summary of immunoblotting results in skeletal muscle and liver after insulin stimulation (Mean values with their standard errors of eight replicates)

\begin{tabular}{|c|c|c|c|c|c|c|}
\hline \multirow[t]{2}{*}{ Diet group†... } & \multicolumn{2}{|c|}{ HFD } & \multicolumn{2}{|c|}{$\csc$} & \multicolumn{2}{|c|}{ NSC } \\
\hline & Mean & SE & Mean & SE & Mean & SE \\
\hline \multicolumn{7}{|l|}{ Relative to GAPDH or tubulin } \\
\hline \multicolumn{7}{|l|}{ Soleus muscle } \\
\hline Total akt & $1 \cdot 18$ & 0.49 & 0.82 & 0.29 & 1.26 & 0.46 \\
\hline Phospho-akt/akt & 0.44 & 0.02 & 0.43 & 0.04 & 0.40 & 0.02 \\
\hline Total AMPK & 0.92 & 0.12 & $1 \cdot 16$ & 0.24 & $1.98^{\star}$ & 0.28 \\
\hline Phospho-AMPK/AMPK & 0.49 & 0.12 & 0.44 & 0.06 & 0.51 & 0.07 \\
\hline \multicolumn{7}{|l|}{ Epitrochlearis muscle } \\
\hline Total akt & 0.60 & 0.07 & $1.08^{*}$ & 0.13 & 0.91 & 0.13 \\
\hline Phospho-akt/akt & 0.58 & 0.08 & 0.58 & $0 \cdot 10$ & 0.61 & 0.09 \\
\hline Total AMPK & 0.57 & 0.19 & 0.86 & 0.19 & 0.56 & 0.13 \\
\hline Phospho-AMPK/AMPK & 0.41 & 0.04 & 0.30 & 0.04 & 0.35 & 0.02 \\
\hline \multicolumn{7}{|l|}{ Relative to $\beta$-actin } \\
\hline \multicolumn{7}{|l|}{ Liver } \\
\hline Total akt & 0.53 & 0.15 & 0.60 & 0.12 & 0.79 & 0.29 \\
\hline Phospho-akt/akt & 0.25 & 0.06 & 0.22 & 0.03 & \multicolumn{2}{|c|}{ NA } \\
\hline Total AMPK & 0.66 & 0.11 & 0.36 & 0.08 & $0.21^{*}$ & 0.03 \\
\hline Phospho-AMPK:AMPK & 0.48 & 0.18 & 0.89 & 0.28 & 1.03 & 0.32 \\
\hline Total PEPCK & 0.25 & 0.03 & 0.20 & 0.03 & 0.24 & 0.03 \\
\hline
\end{tabular}

HFD, high-fat diet; CSC, diet supplemented with coloured seed coats; NSC, diet supplemented with noncoloured seed coats; GAPDH, glyceraldehyde-3-phosphate dehydrogenase; AMPK, AMP-dependent kinase; NA, not available; PEPCK, phosphoenol pyruvate carboxykinase.

* Mean value was significantly different from that of the HFD group $(P<0.05)$.

†The EMB group was omitted from immunoblotting analyses because there was no beneficial effect of this diet on glucose homeostasis. 
(a)

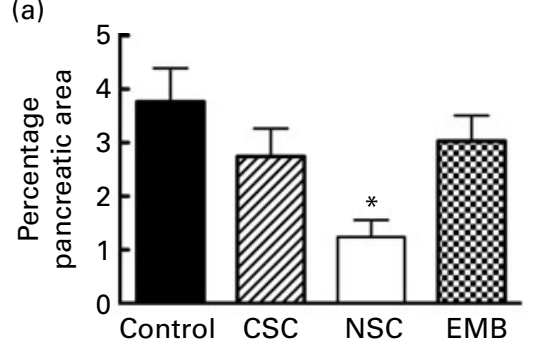

(b)

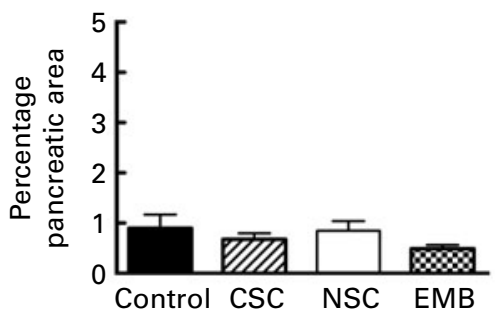

(c)

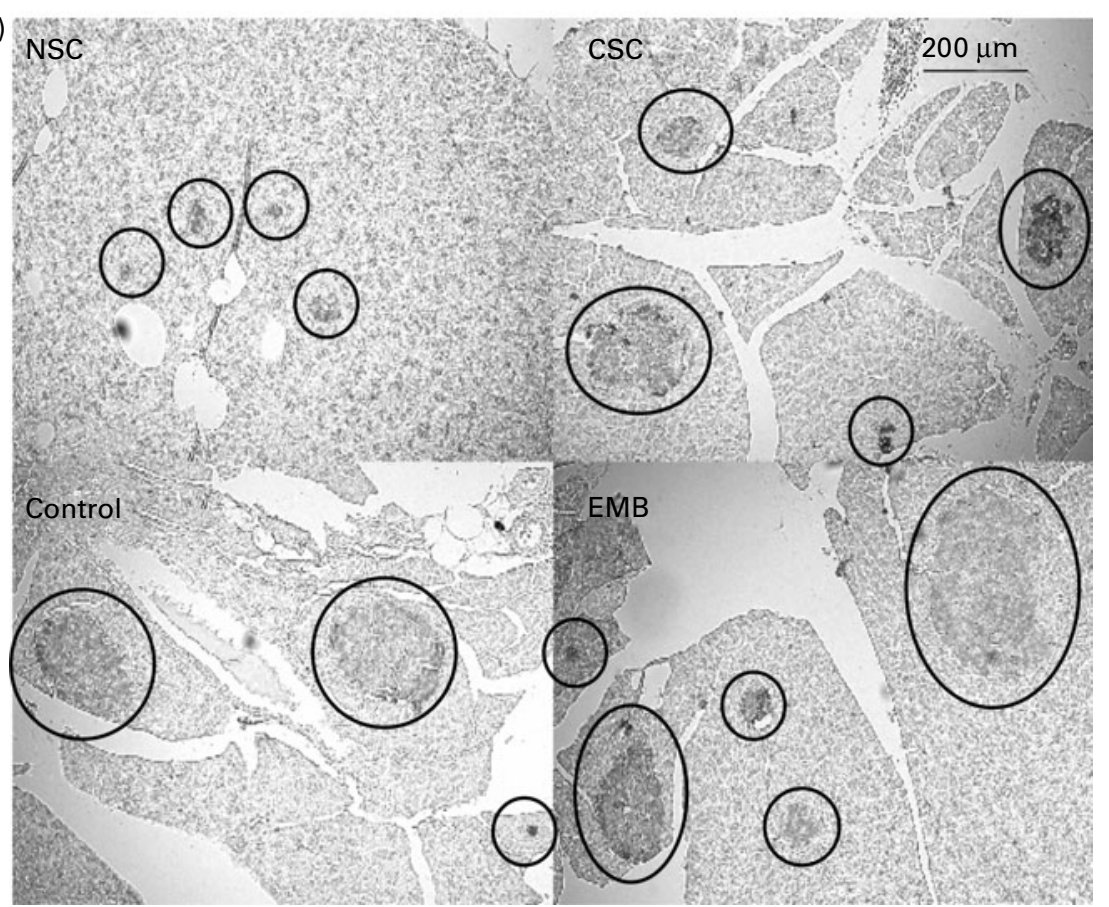

Fig. 3. Morphology of pancreatic islets. The percentage of total pancreatic area occupied by cells immunostaining for (a) insulin and (b) glucagon was quantified. Values are means ( $n 8$ for all groups), with standard errors represented by vertical bars. *Mean value was significantly different from that of the control group fed the high-fat diet $(P<0.05)$. (c) Representative photomicrographs. Insulin immunostaining is highlighted by the circles around each islet. CSC, coloured seed coat diet; NSC, non-coloured seed coat diet; EMB, embryo diet.

higher digestibility than cooked peas, along with lower SCFA concentration and higher $\mathrm{pH}^{(7)}$. The beneficial effects of pea starch found in human studies ${ }^{(8-10)}$ are probably because cooked preparations are used. The benefits of pea seed coats may be attributed to their main nutrient component, acid- and neutral-detergent fibres. In the present study, both the NSC and CSC treatments produced a similar improvement in glucose tolerance but had distinct effects on insulin secretion. More beneficial effects on diverse tissues were detected after feeding NSC. However, the concentration of flavonoids in the CSC diet preparations was quite low.

Both NSC and CSC groups had lower fasting blood glucose after 3 weeks of pea diets and peak glucose excursions in the oral glucose tolerance test were significantly reduced. However, the mechanisms by which NSC and CSC feeding led to improved glucose homeostasis were clearly different, because NSC reduced both fasting insulin and the insulin response to oral glucose, whereas CSC had no effect on insulin. Derivation of HOMA-IR from fasting glucose and insulin values suggests that rats fed NSC had improved insulin sensitivity compared with the HFD or CSC groups. The idea that insulin signalling was improved in NSC rats was consistent with the reduction in insulin secretion and in reduced area of $\beta$-cells in pancreatic tissue. In response to a HFD, $\beta$-cells undergo a rapid hyperplastic response within $10 \mathrm{~d}$ in order to adapt to developing insulin resistance ${ }^{(27)}$; thus, the NSC diet appeared to have reduced the need for this adaptive response. Although these results were obtained from uncooked seed coat preparations, cooking does not greatly affect either insoluble or soluble fibre components ${ }^{(28)}$. Therefore, fibre-dependent effects would probably be retained in cooked preparations more relevant to human consumption. Conversely, cooking alters the chemical structure of polyphenols, which could alter their bioavailability and antioxidant properties ${ }^{(13)}$.

Lower circulating glucose in an oral glucose tolerance test may be observed for several reasons: (a) increased uptake of glucose into peripheral, insulin-sensitive tissues; (b) enhanced suppression of liver glucose output by insulin; or (c) reduced glucose absorption from the gastrointestinal tract. In the present study, differences consistent with 
an increased uptake of glucose were observed in insulinsignalling pathways in skeletal muscle. Skeletal muscle uptake accounts for approximately $75 \%$ of insulin-stimulated glucose disposition. In glycolytic (fast-twitch) muscle of CSC rats (represented by epitrochlearis muscle), total akt protein amount was increased 1-8-fold which, combined with similar relative phosphorylation, could double glucose transport. Akt is one of the critical molecules downstream of phosphatidyl inositol 3-kinase in skeletal muscle that signals GLUT4 translocation and its function is reduced in HFD-induced insulin resistance ${ }^{(29)}$. Dietary manipulation, such as feeding fish protein, has previously been shown to increase akt phosphorylation and improve glucose disposal ${ }^{(30)}$. In the present study, the effects of pea seed coat feeding on akt protein expression were restricted to the CSC, which suggests that the effects were not due to fibre or protein. The CSC contains proanthocyanidins, a class of polyphenols. Other polyphenols such as curcumin and epigallocatechin-3-galate (enriched in green tea) inhibit akt phosphorylation and are touted for anti-cancer effects ${ }^{(31)}$. Anthocyanidins (which are chemically related to proanthocyanidins) from black soyabeans also inhibited akt phosphorylation when applied directly to UVBirradiated cells ${ }^{(32)}$. However, in neurons, flavonoids (a class of polyphenols that includes proanthocyanidins) are reported to increase akt-related signalling, albeit mainly by increasing phosphorylation $^{(33)}$. One mechanism by which a flavonoid may regulate total akt protein expression is by interactions with heat-shock proteins (Hsp). Hsp70 attenuates akt expression; its inhibition by the flavonoid myricetin led to an increase in akt protein in a breast cancer cell line ${ }^{(34)}$. (a)
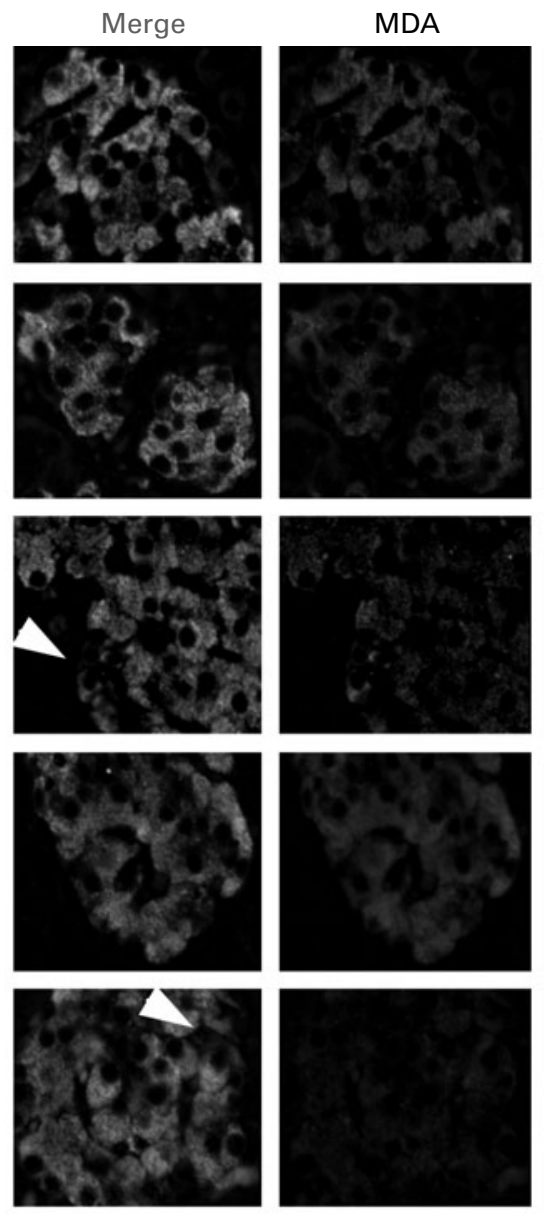

(b)
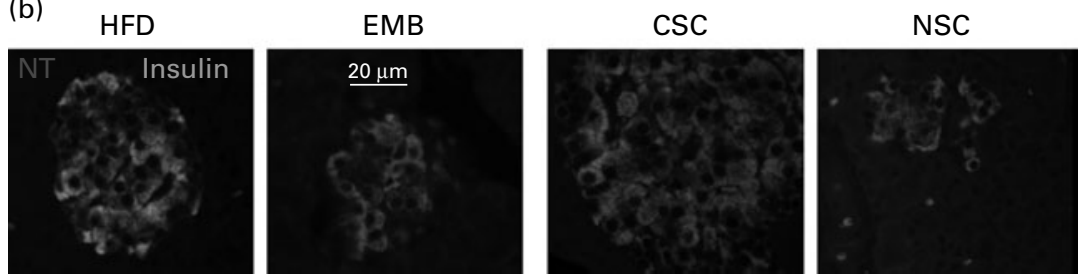

Fig. 4. Assessment of oxidative stress in the islets by dual immunostaining for insulin and malondialdehyde (MDA) (a) and insulin and nitrotyrosine (NT) (b). MDA co-localised with cells labelled for insulin in all groups, and with cells not labelled for insulin only in the non-coloured seed coat (NSC) group ( $\rightarrow$ ). NT staining was limited to cells not staining for insulin in all groups. LFD, low-fat diet; HFD, high-fat diet; CSC, coloured seed coat diet; EMB, embryo diet. 
(a)

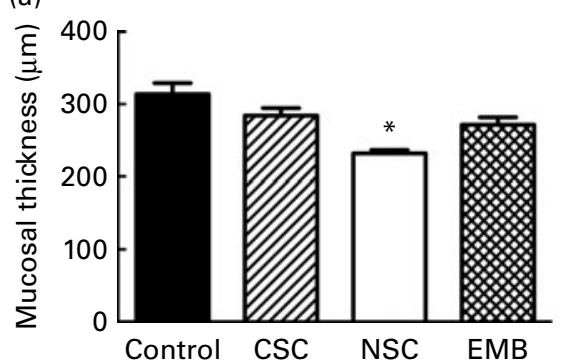

(b)

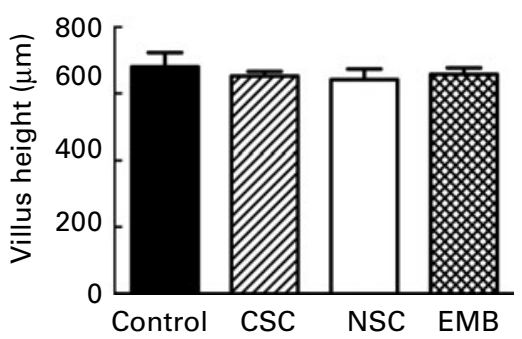

Fig. 5. Effects of feeding pea fractions on gut morphology. (a) Thickness of the colonic mucosa. (b) Villus height in the jejunum. Values are means ( $n 8$ for all groups), with standard errors represented by vertical bars. *Mean value was significantly reduced compared with the other groups $(P<0.05)$. No effects of pea fractions on villus height in the jejunum were detected. CSC, coloured seed coat diet; NSC, non-coloured seed coat diet; EMB, embryo diet.

Whether this is the mechanism by which CSC regulate akt activity requires further experimentation.

Adaptations in akt protein expression levels or phosphorylation were not seen in the liver or soleus (oxidative) muscle. However, in the soleus muscle of NSC rats, total AMPK protein expression was elevated, which could contribute to noninsulin-regulated glucose uptake and the lower fasting glucose concentrations. Previously, Liu et al. ${ }^{(35)}$ reported that HFD feeding reduced levels of both total and phosphorylated AMPK. Thus, the increase after NSC feeding could represent normalisation of AMPK levels, although not phosphorylation. Unexpectedly, in the liver, total AMPK protein expression was reduced by the NSC diet but, combined with a nonsignificant increase in phospho-AMPK, the impact on overall metabolism is predicted to be small. To address the possibility that lower blood glucose was secondary to reduced hepatic glucose output the amount of PEPCK, the major regulatory enzyme of gluconeogenesis, was examined but no differences were detected between the groups.

Improved glucose tolerance and lower insulin secretion in the NSC group could also be explained by slower or reduced glucose absorption from the gastrointestinal tract. Assessment of the absorptive area of the jejunum and colon was conducted. The height of the villi was taken as an index of absorptive area in the jejunum and was not different between the groups. In the colon, the thickness of the mucosa was decreased in the NSC group. In rats, carrier-mediated glucose absorption may occur in the colon although with slower kinetics than in the jejunum ${ }^{(36)}$. GLUT2, GLUT5 and the Na-dependent GLUT SGLT1 were all detectable by immunostaining in the colonic mucosa of all rat groups, concentrated in the epithelial cells at the luminal boundary (data not shown). However, other mechanisms of glucose uptake involving hydrogen ion movement may also be active ${ }^{(37)}$. Furthermore, soluble fibre from other legume seeds (fenugreek) inhibited intestinal disaccharidase activity and increased gastrointestinal motility, which could also contribute to a reduced absorption of glucose ${ }^{(38)}$. Of interest is a study showing that SGLT1 can transport the flavonoid quercetin ${ }^{(39)}$ and inhibit glucose transport in a swine model ${ }^{(40)}$. These potential mechanisms were not investigated in the present study.

A benefit of feeding pea NSC was reduced stress on pancreatic islet cells. Feeding a HFD is well known to induce $\beta$-cell proliferation, with an overall expansion of $\beta$-cell mass $^{(27)}$. The degree of proliferation may depend upon the severity of hyperglycaemia because glucose stimulates $\beta$-cell mitosis $^{(41)}$. Conversely, a HFD also induce apoptosis and the degree to which islets enlarge is determined by cell proliferation minus cell death ${ }^{(42)}$. NSC-fed rats had smaller islets than rats in any other group. We conclude that reduced islet size is not due to increased apoptosis because the structure of the islets was otherwise normal, and both $\beta$ - and $\alpha$-cells stained less intensely for MDA, which is formed as a result of lipid peroxidation and a marker of oxidative stress ${ }^{(43)}$. We also assessed NT staining in islets. Nitration of tyrosine in proteins occurs when $\mathrm{NO}$ and superoxide react together ${ }^{(44)}$. The present results suggest the effects of feeding NSC involve a reduction of lipid peroxidation but not production of NT. Although flavonoids from various sources and of several chemical classes have previously been shown to have both beneficial (for example, increased response to glucose) or detrimental (for example, increased apoptosis) effects ${ }^{(45)}$, the present results suggest that flavonoids in the CSC group did not have any effect on $\beta$-cells.

\section{Conclusions}

Diets containing uncooked pea seed coats but not the embryos had beneficial effects on glucose tolerance in rats fed a HFD. NSC also reduced insulin secretion and this was associated with reduced expansion of the $\beta$-cell mass and MDA production, indicating reduced glycaemic stress on the islets. This outcome is potentially important because type 2 diabetes is caused by both insulin resistance and $\beta$-cell dysfunction, with the failure of the $\beta$-cells to adapt ultimately precipitating hyperglycaemia $^{(46)}$. The mechanisms by which pea seed coats exert their effects on blood glucose were not definitely identified but appeared to involve insulin signalling in skeletal muscle.

\section{Acknowledgements}

The present study was funded by grants from Alberta Innovates BioSolutions and the Alberta Pulse Growers Commission. Neither funder placed any restrictions on publication of the present study. K. A. W. and L. K. received stipend support from Alberta Innovates Health Solutions. The authors are grateful to D. O'Neill and Z. Fatehi-Hassanabad for technical 
support. C. B. C., C. J. F., R. C. B. and J. A. O. conceived of and designed the experiments. K. A. W., L. K., H. Y., C. H. and J. M. conducted the experiments and statistical analysis. A. J. prepared the pea fractions and performed seed fraction analysis. All authors participated in data interpretation. C. B. C. wrote the manuscript with input from K. A. W., C. H. and J. M., and C. J. F., R. C. B. and J. A. O. edited it. None of the authors reports any conflicts of interest.

\section{References}

1. Canadian Diabetes Association (2008) Clinical practice guidelines for the prevention and management of diabetes in Canada. Can J Diabetes 32, Suppl. 1, S1-S200.

2. Sievenpiper J, Kendall C, Esfahani A, et al. (2009) Effect of non-oil-seed pulses on glycaemic control: a systematic review and meta-analysis of randomised controlled experimental trials in people with and without diabetes. Diabetologia 52, 1479-1495.

3. Ayele BT, Ozga JA \& Reinecke DM (2006) Regulation of GA biosynthesis genes during germination and young seedling growth of pea (Pisum sativum). J Plant Growth Regul 25, 219-232.

4. Guillon F \& Champ MM (2002) Carbohydrate fractions of legumes: uses in human nutrition and potential for health. Br J Nutr 88, Suppl. 3, S293-S306.

5. Daveby YD, Abrahamsson M \& Aman P (1993) Changes in chemical composition during development of three different types of peas. J Sci Food Agric 63, 21-28.

6. Rochfort S \& Panozzo J (2007) Phytochemicals for health, the role of pulses. J Agric Food Chem 55, 7981-7994.

7. Goodlad JS \& Mathers JC (1992) Digestion of complex carbohydrates and large bowel fermentation in rats fed on raw and cooked peas (Pisum sativum). Br J Nutr 67, 475-488.

8. Chandalia M, Garg A, Lutjohann D, et al. (2000) Beneficial effects of high dietary fiber intake in patients with type 2 diabetes mellitus. N Engl J Med 342, 1392-1398.

9. Higgins JA (2004) Resistant starch: metabolic effects and potential health benefits. $J A O A C$ Int $\mathbf{8 7}, 761-768$.

10. Weickert MO \& Pfeiffer AFH (2008) Metabolic effects of dietary fiber consumption and prevention of diabetes. $J$ Nutr 138, 439-442.

11. Xu BJ, Yuan SH \& Chang SK (2007) Comparative analyses of phenolic composition, antioxidant capacity, and color of cool season legumes and other selected food legumes. J Food Sci 72, S167-S177.

12. Duenas M, Estrella I \& Hernandez T (2002) Occurrence of phenolic compounds in the seed coat and the cotyledon of peas (Pisum sativum L.). Eur Food Res Tech 219, 116-123.

13. Serrano J, Puupponen-Pimiä R, Dauer A, et al. (2009) Tannins: current knowledge of food sources, intake, bioavailability and biological effects. Mol Nutr Food Res 53, Suppl. 2, S310-S329.

14. Vislocky LM \& Fernandez ML (2010) Biomedical effects of grape products. Nutr Rev 68, 656-670.

15. Lee YA, Cho EJ \& Yokozawa T (2008) Effects of proanthocyanidin preparations on hyperlipidemia and other biomarkers in mouse model of type 2 diabetes. J Agric Food Chem 56, 7781-7789.

16. Schafer G, Schenk U, Ritzel U, et al. (2003) Comparison of the effects of dried peas with those of potatoes in mixed meals on postprandial glucose and insulin concentrations in patients with type 2 diabetes. Am J Clin Nutr 78, 99-103.

17. Marinangeli CP \& Jones PJ (2010) Whole and fractionated yellow pea flours reduce fasting insulin and insulin resistance in hypercholesterolaemic and overweight human subjects. Br J Nutr 105, 110-117.

18. Seewi G, Gnauck G, Stute R, et al. (1999) Effects on parameters of glucose homeostasis in healthy humans from ingestion of leguminous versus maize starches. Eur J Nutr 38, 183-189.

19. Sandstrom B, Hansen LT \& Sorensen A (1994) Pea fiber lowers fasting and postprandial blood triglyceride concentrations in humans. J Nutr 124, 2386-2396.

20. AOAC International (2000) AOAC Official Method 972.43: Micro-Chemical Determination of Carbon, Hydrogen, and Nitrogen, Automated Method, 17th ed. Arlington, VA: AOAC International.

21. ANKOM Technology (2006) ANKOM Technology Method 6: Neutral Detergent Fiber in Feeds Filter Bag Technique. Macedon, NY: ANKOM Technology.

22. ANKOM Technology (2006) ANKOM Technology Method 5: Acid Detergent Fiber in Feeds Filter Bag Techniques. Macedon, NY: ANKOM Technology.

23. Kennedy JA \& Jones GP (2001) Analysis of proanthocyanidin cleavage products following acid-catalysis in the presence of excess phloroglucinol. J Agric Food Chem 49, 1740-1746.

24. Nikolopoulou D, Grigorakis K, Stasini M, et al. (2007) Differences in chemical composition of field pea (Pisum sativum) cultivars: effects of cultivation area and year. Food Chem 103, 847-852.

25. Chan CB, Pederson RA, Buchan AM, et al. (1984) Gastric inhibitory polypeptide (GIP) and insulin release in the obese Zucker rat. Diabetes 33, 536-542.

26. Saleh MC, Fatehi-Hassanabad Z, Wang R, et al. (2008) Mutated ATP synthase induces oxidative stress and impaired insulin secretion in $\beta$-cells of female BHE/cdb rats. Diabetes/ Metab Res Rev 24, 392-403.

27. Smith SJ, Zhang $\mathrm{H}$, Clermont $\mathrm{AO}$, et al. (2006) In vivo monitoring of pancreatic $\beta$-cells in a transgenic mouse model. Mol Imaging 5, 66-75.

28. de Almeida Costa GE, da Silva Queiroz-Monici K, Machado Reis SMP, et al. (2006) Chemical composition, dietary fibre and resistant starch contents of raw and cooked pea, common bean, chickpea and lentil legumes. Food Chem 94, 327-330.

29. Tremblay F, Lavigne C, Jacques H, et al. (2001) Defective insulin-induced GLUT4 translocation in skeletal muscle of high-fat-fed rats is associated with alterations in both Akt/ PKB and atypical PKC $(\zeta / \lambda)$ activities. Diabetes 50 , 1901-1910.

30. Tremblay F, Lavigne C, Jacques H, et al. (2003) Dietary cod protein restores insulin-induced activation of phosphatidylinositol 3-kinase/Akt and GLUT4 translocation to the T-tubules in skeletal muscle of high-fat-fed obese rats. Diabetes 52, 29-37.

31. Lamoral-Theys D, Pottier L, Dufrasne F, et al. (2010) Natural polyphenols that display anticancer properties through inhibition of kinase activity. Curr Med Chem 17, 812-825.

32. Tsoyi K, Park HB, Kim YM, et al. (2008) Anthocyanins from black soybean seed coats inhibit UVB-induced inflammatory cylooxygenase-2 gene expression and PGE2 production through regulation of the nuclear factor- $\kappa \mathrm{B}$ and phosphatidylinositol 3-kinase/Akt pathways. J Agric Food Chem 56, 8969-8974.

33. Spencer JPE (2007) The interactions of flavonoids within neuronal signalling pathways. Genes Nutr 2, 257-273.

34. Koren J III, Jinwal UK, Jin Y, et al. (2010) Facilitating Akt clearance via manipulation of Hsp70 activity and levels. J Biol Chem 285, 2498-2505. 
35. Liu Y, Wan Q, Guan Q, et al. (2005) High-fat diet feeding impairs both the expression and activity of AMPK $\alpha$ in rats skeletal muscle. Biochem Biophys Res Commun 339, 701-717.

36. Tomei S, Torimoto M, Hayashi Y, et al. (2003) Kinetic characterization of carrier-mediated transport systems for d-glucose and taurocholate in the everted sacs of the rat colon. Biol Pharm Bull 26, 899-901.

37. Yamamoto F, Nakada K, Zhao S, et al. (2004) Gastrointestinal uptake of FDG after N-butylscopolamine or omeprazole treatment in the rat. Ann Nucl Med 18, 637-640.

38. Hannan JM, Ali L, Rokeya B, et al. (2007) Soluble dietary fibre fraction of Trigonella foenum-graecum (fenugreek) seed improves glucose homeostasis in animal models of type 1 and type 2 diabetes by delaying carbohydrate digestion and absorption, and enhancing insulin action. $\mathrm{BrJ}$ Nutr 97, 514-521.

39. Wolffram S, Block M \& Ader P (2002) Quercetin-3-glucoside is transported by the glucose carrier SGLT1 across the brush border membrane of the rat small intestine. J Nutr 132, $130-135$.
40. Cermak R, Landgraf S \& Wolffram S (2004) Quercetin glucosides inhibit glucose uptake into brush-border-membrane vesicles of porcine jejunum. Br J Nutr 91, 849-855.

41. Bonner-Weir S, Deery D, Leahy JL, et al. (1989) Compensatory growth of pancreatic $\beta$-cells in adult rats after short-term glucose infusion. Diabetes 38, 49-53.

42. Bonner-Weir S (2000) Life and death of the pancreatic $\beta$ cells. Trends Endocrinol Metab 11, 375-378.

43. Trevisan R, Browne R, Ram M, et al. (2001) Correlates of markers of oxidative status in the general population. Am J Epidemiol 154, 348-356.

44. Schopfer FJ, Baker PR \& Freeman BA (2003) NO-dependent protein nitration: a cell signaling event or an oxidative inflammatory response? Trends Biochem Sci 28, 646-654.

45. Pinent M, Castell A, Baiges I, et al. (2008) Bioactivity of flavonoids on insulin-secreting cells. Comp Rev Food Sci Food Safety 7, 299-308.

46. LeRoith D (2002) $\beta$-Cell dysfunction and insulin resistance in type 2 diabetes: role of metabolic and genetic abnormalities. Am J Med 113, Suppl. 6A, 3S-11S. 